\title{
A NON-DC FUNCTION WHICH IS DC ALONG ALL CONVEX CURVES
}

\author{
LIBOR VESELÝ AND LUDĚK ZAJÍČEK
}

\begin{abstract}
A problem asked by the authors in 1989 concerns the natural question, whether one can deduce that a continuous function $f$ on an open convex set $D \subset \mathbb{R}^{n}$ is DC (i.e., is a difference of two convex functions) from the behavior of $f$ "along some special curves $\varphi$ ". I.M. Prudnikov published in 2014 a theorem (working with convex curves $\varphi$ in the plane), which would give a positive answer in $\mathbb{R}^{2}$ to our problem. However, in the present note we construct an example showing that this theorem is not correct, and thus our problem remains open in each $\mathbb{R}^{n}, n>1$.
\end{abstract}

\section{INTRODUCTION}

A function $f$ on an open convex set $D \subset \mathbb{R}^{n}$ is called a $\mathrm{DC}$ (or d.c.) function if it is a difference of two convex functions. Fore more information about DC functions and their applications see e.g. [3], [6], [1].

DC functions of one variable have a very simple internal characterization: they are precisely indefinite integrals of functions with locally bounded variation. However, for $n \geq 2$, no simple and useful internal characterization of DC functions is known.

Already A.D. Aleksandrov (who first studied and used DC functions of more variables) in 1949 asked whether a function on $\mathbb{R}^{2}$ which is (in a natural sense) DC on each line must be DC; now it is well-known that this is not the case (see e.g. [7, p. 35]). So the following rough question arises:

Is it possible to characterize DC functions on $D \subset \mathbb{R}^{n}$ "in the language of curves" only?

Two precise versions of this question were formulated in [7, Problems 6 and 7, p. 45]; Problem 7 is reproduced in Remark 2.2 below. A paper [4] by I.M. Prudnikov contains a claim (Theorem 1 ) which would give a positive answer to [7, Problem 7] for real functions in $\mathbb{R}^{2}$ (see Remark 2.2). However, we construct an example showing that $[4$, Theorem 1] is not correct, and thus our [7, Problem 7] remains open in each $\mathbb{R}^{n}, n>1$, even for real functions.

Let us describe the claim of [4, Theorem 1$]$ :

Date: March 6, 2018.

2000 Mathematics Subject Classification. 26B25; 26B40.

Key words and phrases. DC function, d.c. function, characterization of DC functions. 
For a two-dimensional compact convex set $K \subset \mathbb{R}^{2}$ let $r_{K}:\left[0, T_{K}\right] \rightarrow \partial K$ be a simple closed curve parametrized by the arc length. Let $D \subset \mathbb{R}^{2}$ be an open bounded convex set and $f: D \rightarrow \mathbb{R}$ a Lipschitz function.

[4, Theorem 1] asserts, that $f$ is DC if and only if the following condition holds:

(C) There exists a constant $c=c(D, f)>0$ such that for each twodimensional convex compact $K \subset D$ the variation $V\left(\Phi^{\prime},\left[0, T_{K}\right]\right)$ is less than $c$, where $\Phi(t):=f\left(r_{K}(t)\right), t \in\left[0, T_{K}\right]$. (The variation is calculated by using only partitions consisting of points of differentiability of $\Phi$.)

(Note that the assumption of convexity of $K$ is omitted in the English translation of [4].)

The main aim of the present note is to show (by a counterexample) that condition (C) does not imply that $f$ is DC. It is difficult to specify which step in the proof of [4, Theorem 1] is incorrect, since many arguments in this proof are sketched only.

\section{Preliminaries}

We consider $\mathbb{R}^{d}$ equipped with the standard Euclidean norm $\|\cdot\|$. Given $x \in \mathbb{R}^{d}$ and $r>0$, by $B(x, r)$ we denote the closed ball of center $x$ and radius $r$.

Let $I \subset \mathbb{R}$ be a non-degenerate interval, and $h: I \rightarrow \mathbb{R}^{d}$. If $I$ is compact the variation $V(h, I)$ of $h$ on $I$ is defined in the standard way via finite partitions of $I$. For an arbitrary $I$, we put

$$
V(h, I)=\sup \{V(h,[a, b]): a, b \in I, a<b\} .
$$

It is elementary to see that we always have

(1)

$$
\begin{aligned}
V(h,[a, b]) & \leq V(h,[a, b))+\limsup _{s \rightarrow b^{-}}\|h(s)-h(b)\| \\
& \leq V(h,(a, b))+\limsup _{s \rightarrow a^{+}}\|h(s)-h(a)\|+\limsup _{s \rightarrow b^{-}}\|h(s)-h(b)\| .
\end{aligned}
$$

Let us recall the following definition from [2]. Given a continuous mapping $F:[a, b] \rightarrow \mathbb{R}^{d}$ and a finite partition $D=\left\{a=t_{0}<t_{1}<\cdots<t_{n}=b\right\}$ of $[a, b]$, we define

$$
P(F, D):=\sum_{i=1}^{n-1}\left\|\frac{F\left(t_{i+1}\right)-F\left(t_{i}\right)}{\left\|F\left(t_{i+1}\right)-F\left(t_{i}\right)\right\|}-\frac{F\left(t_{i}\right)-F\left(t_{i-1}\right)}{\left\|F\left(t_{i}\right)-F\left(t_{i-1}\right)\right\|}\right\|
$$


if the quantity of the right-hand side makes sense, otherwise $P(F, D):=0$. The quantity

$$
P_{a}^{b} F:=\sup _{D} P(F, D)
$$

is called the turn of $F$ on $[a, b]$.

Analogously, the convexity of $F$ over $[a, b]$ is the quantity

$$
K_{a}^{b} F:=\sup _{D} K(F, D)
$$

where

$$
K(F, D):=\sum_{i=1}^{n-1}\left\|\frac{F\left(t_{i+1}\right)-F\left(t_{i}\right)}{t_{i+1}-t_{i}}-\frac{F\left(t_{i}\right)-F\left(t_{i-1}\right)}{t_{i}-t_{i-1}}\right\| .
$$

We shall sometimes use the alternative notation $K(F,[a, b]):=K_{a}^{b} F$.

Lemma 1.1. Let $K \subset \mathbb{R}^{2}$ be a two-dimensional compact convex set, and $r:[0, \ell] \rightarrow \partial K$ a parametrization by the arc-length of $\partial K$ as a simple closed curve. Then $r$ admits the right derivative $r_{+}^{\prime}(t)$ at each $t \in[0, \ell)$, and the left derivative $r_{-}^{\prime}(t)$ at each $t \in(0, \ell]$. Moreover,

$$
K_{0}^{\ell} r=P_{0}^{\ell} r \leq 2 \pi
$$

Proof. We can (an do) assume that $r$ is counterclockwise oriented. Given a partition $D=\left\{0=t_{0}<t_{1}<\cdots<t_{n}=\ell\right\}$, convexity of $K$ implies that the vectors $v_{i}:=\frac{r\left(t_{i}\right)-r\left(t_{i-1}\right)}{\left\|r\left(t_{i}\right)-r\left(t_{i-1}\right)\right\|}, i=1, \ldots, n$, are "ordered in the counterclockwise way" on the unit circumference in the plane, and $P(r, D)$ is the length of the corresponding simple (not necessarily closed) polygonal curve inscribed in the unit circle. Thus clearly $P_{0}^{\ell} r \leq 2 \pi$ (= the length of the unit circumference). By [2, Theorem 4.10], $r$ has finite "turn of tangents" $T_{0}^{\ell} r$ (for definition see $[2$, pp. 25-26]) which satisfies $T_{0}^{\ell} r=P_{0}^{\ell} r$. By [2, Lemma 4.4], $r$ admits at each point both one-sided derivatives $r_{ \pm}^{\prime}(t)$ and they are equal to the corresponding "one-sided tangents" $\tau_{ \pm}(r, t)$ (for definition see $[2$, p. 25]). Finally, by $[2$, Proposition 5.7], $r$ has finite convexity and $K_{0}^{\ell} r=T_{0}^{\ell} r=P_{0}^{\ell} r \leq 2 \pi$ holds.

\section{The COUnterexample}

There exists a Lipschitz real-valued function $f$ on $D:=(-2,2)^{2}$ such that $f$ is not $D C$ and satisfies condition $(C)$.

Our construction will proceed in three steps. In the first step we will construct a mapping ("curve") $\varphi: \mathbb{R} \rightarrow D$ which is DC, in the sense that all its components are DC functions. The second step will produce a Lipschitz function $f: D \rightarrow \mathbb{R}$ such that $f \circ \varphi$ is not DC (which implies that $f$ is not DC). In the third, most difficult step, we will prove that condition (C) is satisfied. 
First step: construction of $\varphi$.

Choose a sequence $a_{n}>0, n \in \mathbb{N}$, with $\sum_{n=1}^{\infty} a_{n}=1 / 2$. Then we can clearly choose $h_{n}>0, n \in \mathbb{N}$, such that $\sum_{n=1}^{\infty} h_{n}=1$, and $\sum_{n=1}^{\infty} h_{n} / a_{n}<\infty$.

Further set $b_{1}:=0, b_{n}:=2 \sum_{i=1}^{n-1} a_{i}$ for $n \geq 2$ and $c_{n}:=b_{n}+a_{n}, n \in \mathbb{N}$. Thus $0=b_{1}<c_{1}<b_{2}<c_{2}<\cdots<1, \lim b_{n}=\lim c_{n}=1$ and $c_{n}-b_{n}=$ $b_{n+1}-c_{n}=a_{n}, n \in \mathbb{N}$.

Let $g$ be the (unique) function on $\mathbb{R}$ such that (i) $g\left(b_{1}\right)=0, g\left(c_{1}\right)=0, g\left(b_{n}\right)=g\left(c_{n}\right)=\sum_{i=1}^{n-1} h_{i}$ for $n \geq 2, g(1)=$
$\sum_{i=1}^{\infty} h_{i}=1$,

(ii) $g$ is affine on each $\left[b_{n}, c_{n}\right]$ and $\left[c_{n}, b_{n+1}\right], n \in \mathbb{N}$, and

(iii) $g$ is constant on $(-\infty, 0]$ and $[1, \infty)$.

Set

$$
\varphi(t):= \begin{cases}(t, g(t)) & \text { for } t \in[0,1] \\ (0,0) & \text { for } t<0 \\ (1,1) & \text { for } t>1\end{cases}
$$

Obviously, $g$ is nondecreasing and $\varphi(\mathbb{R}) \subset D$. Since $\sup \left\{h_{n} / a_{n}: n \in \mathbb{N}\right\}<\infty$, it is easy to see that both $g$ and $\varphi$ are Lipschitz. Further, the right derivative $\varphi_{+}^{\prime}(t)$ equals: $(1,0)$ for $t \in\left[b_{n}, c_{n}\right) ;\left(1, h_{n} / a_{n}\right)$ for $t \in\left[c_{n}, b_{n+1}\right)$; and $(0,0)$ for $t \notin[0,1)$. Clearly $\lim _{s \rightarrow 1^{-}} \varphi_{+}^{\prime}(s)=(1,0)$ since $h_{n} / a_{n} \rightarrow 0$. Now, (1) and the definition of $\varphi$ easily yield

$$
V\left(\varphi_{+}^{\prime}, \mathbb{R}\right) \leq V\left(\varphi_{+}^{\prime},[0,1)\right)+\|(1,0)\|=2 \sum_{n=1}^{\infty} h_{n} / a_{n}+1<\infty .
$$

By [7, Theorem 2.3] we obtain that $\varphi$ is DC.

\section{Second step: construction of $f$.}

Let $t_{0}^{(n)}=b_{n}<t_{1}^{(n)}<t_{2}^{(n)}<\cdots<t_{n}^{(n)}<c_{n}=t_{n+1}^{(n)}$ be an equidistant partition of the interval $\left[b_{n}, c_{n}\right](n \in \mathbb{N})$; so $t_{j+1}^{(n)}-t_{j}^{(n)}=\left(c_{n}-b_{n}\right) /(n+1)=a_{n} /(n+1)$.

Choose $r_{n}>0, n \in \mathbb{N}$, so small that

$$
\begin{gathered}
r_{n}<\frac{a_{n}}{4(n+1)}, r_{n}<\frac{h_{n}}{2} \text { and } r_{n}<\frac{h_{n-1}}{2}(n \geq 2), \\
r_{n}+\frac{4 r_{n}(n+1)}{a_{n}}\left(1-b_{n}\right)<\frac{h_{n}}{2}, \text { and } \\
-r_{n}-\frac{4 r_{n}(n+1)}{a_{n}} c_{n}>-\frac{h_{n-1}}{2}, \quad n \geq 2 .
\end{gathered}
$$


Now, for $n \in \mathbb{N}$ and $1 \leq j \leq n$, set $z_{j}^{(n)}:=\varphi\left(t_{j}^{(n)}\right)=\left(t_{j}^{(n)}, g\left(t_{j}^{(n)}\right)\right)$. The first inequality of (2) implies that the balls

$$
B_{j}^{(n)}:=B\left(z_{j}^{(n)}, r_{n}\right) \quad(n \in \mathbb{N}, 1 \leq j \leq n)
$$

are pairwise disjoint subsets of $D$, and so we can define $f: D \rightarrow \mathbb{R}$ by

$$
f(x):= \begin{cases}\frac{1}{n^{2}}\left(r_{n}-\left\|x-z_{j}^{(n)}\right\|\right) & \text { if } x \in B_{j}^{(n)}, \\ 0 & \text { if } x \in D \backslash \bigcup_{n=1}^{\infty} \bigcup_{j=1}^{n} B_{j}^{(n)} .\end{cases}
$$

It is easy to see that $f$ is Lipschitz with constant 1 . Put $F(t):=f(\varphi(t))$, $t \in \mathbb{R}$, and notice that $F\left(t_{j}^{(n)} \pm r_{n}\right)=0, F\left(t_{j}^{(n)}\right)=r_{n} / n^{2}$, and $F$ is affine on $\left[t_{j}^{(n)}-r_{n}, t_{j}^{(n)}\right]$ and $\left[t_{j}^{(n)}, t_{j}^{(n)}+r_{n}\right](n \in \mathbb{N})$. Now (supposing that $F_{+}^{\prime}$ exists on $\mathbb{R})$, we have

$$
\begin{aligned}
V\left(F_{+}^{\prime},[0,1]\right) & \geq \sum_{n=1}^{\infty} V\left(F_{+}^{\prime},\left[b_{n}, c_{n}\right]\right) \geq \sum_{n=1}^{\infty} \sum_{j=1}^{n}\left|F_{+}^{\prime}\left(t_{j}^{(n)}-r_{n}\right)-F_{+}^{\prime}\left(t_{j}^{(n)}\right)\right| \\
& \geq \sum_{n=1}^{\infty} n \cdot \frac{2\left(r_{n} / n^{2}\right)}{r_{n}}=\infty
\end{aligned}
$$

So, [7, Theorem 2.3] implies that $F$ is not DC. Consequently $f$ is not DC either (see e.g. [7, Theorem 4.2 and Theorem 1.20]).

\section{Third step: proof of (C).}

In the sequel we will essentially use the following proposition which is a special case of [8, Theorem 4.1].

Proposition 2.1. Let $D \subset \mathbb{R}^{n}$ be an open convex set and let $f$ be a nonconstant DC function on D which admits a Lipschitz control function $\gamma$. Let $\varphi:[\alpha, \beta] \rightarrow D$ be Lipschitz. Then

$$
K_{\alpha}^{\beta}(f \circ \varphi) \leq(\operatorname{Lip} f+\operatorname{Lip} \gamma) K_{\alpha}^{\beta} \varphi+2 \operatorname{Lip} \gamma \operatorname{Lip} \varphi .
$$

Here the notion of a control function is used, which is essential in the theory of DC mappings (see [7]). If $f$ is a real DC function then $\gamma$ is a control function for $f$ iff both $\pm f+\gamma$ are convex. So,

(5) if $f=g-h$, with $g, h$ convex, then $g+h$ is a control function for $f$.

Remark 2.2. Proposition 2.1 naturally motivates the following problem, which is a special case of [7, Problem 7].

Problem. Let $D \subset \mathbb{R}^{n}$ be an open convex set and let $f: D \rightarrow \mathbb{R}$ be a function. Suppose that there are $a \geq 0, b \geq 0$ such that

$$
K_{\alpha}^{\beta}(f \circ \varphi) \leq a K_{\alpha}^{\beta} \varphi+b \operatorname{Lip} \varphi
$$


whenever $\varphi:[\alpha, \beta] \rightarrow D$ is Lipschitz. Is then $f$ DC on $D$ ?

Let us note that if $n=2$ and $a, b$ from the above problem exist, then condition (C) from the introduction holds with $C(D, f)=2 \pi a+b$. Indeed, using Proposition 2.1 with $\varphi(t)=r_{K}(t), t \in[\alpha, \beta]=\left[0, T_{K}\right]$, we obtain for $\Phi=f \circ r_{K}$

$$
K_{\alpha}^{\beta} \Phi \leq a K_{\alpha}^{\beta} r_{K}+b \operatorname{Lip} r_{K} \leq 2 \pi a+b
$$

by Lemma 1.1 and 1-lipschitzness of $r_{K}$. So $K_{\alpha}^{\beta} \Phi<\infty$, and therefore $K_{\alpha}^{\beta} \Phi=$ $V\left(\Phi_{+}^{\prime},[\alpha, \beta)\right.$ ) (see, e.g., $\left[8\right.$, Theorem 3.1]). Using (1) and the equality $\Phi_{-}^{\prime}(\beta)=$ $\lim _{t \rightarrow \beta-} \Phi_{+}^{\prime}(t)$ (see, e.g., [8, Proposition 3.4]), we obtain

$$
V\left(\Phi^{\prime},\left[0, T_{K}\right]\right) \leq V\left(\Phi_{+}^{\prime},[\alpha, \beta)\right) \leq 2 \pi a+b .
$$

So, if $[4$, Theorem 1] were correct, the above problem would have a positive answer for $n=2$.

We continue with the following claim.

Claim 1. Let $[u, v] \subset(-2,2)$, and let $h:[u, v] \rightarrow(-2,2)$ be a continuous function which is convex or concave. Then there exists at most one $n \in \mathbb{N}$ such that the graph of $h$ intersects both $B_{j}^{(n)}$ and $B_{k}^{(n)}$ for some $1 \leq j<k \leq n$.

Proof of Claim 1. Assume that the assertion of the claim is false. First, let $h$ be convex. Then we can choose $1 \leq m<n, 1 \leq j<k \leq n, 1 \leq l \leq m$ and $x_{1}, x_{2}, x \in[u, v]$ such that

$$
\left(x_{1}, h\left(x_{1}\right)\right) \in B_{j}^{(n)},\left(x_{2}, h\left(x_{2}\right)\right) \in B_{k}^{(n)} \text { and }(x, h(x)) \in B_{l}^{(m)} .
$$

Clearly $b_{n}<x_{1}<x_{2}<c_{n}$ and $b_{m}<x<c_{m}$. Using convexity of $h$, we obtain

$$
\begin{aligned}
& \frac{\left(g\left(c_{n}\right)-r_{n}\right)-h(x)}{x_{1}-x} \leq \frac{h\left(x_{1}\right)-h(x)}{x_{1}-x} \leq \frac{h\left(x_{2}\right)-h\left(x_{1}\right)}{x_{2}-x_{1}} \\
& \quad \leq \frac{\left(g\left(b_{n}\right)+r_{n}\right)-\left(g\left(b_{n}\right)-r_{n}\right)}{\frac{a_{n}}{2(n+1)}}=\frac{4(n+1) r_{n}}{a_{n}},
\end{aligned}
$$

and hence

$$
g\left(c_{n}\right)-r_{n}-h(x) \leq \frac{4(n+1) r_{n}}{a_{n}}\left(x_{1}-x\right) \leq \frac{4(n+1) r_{n}}{a_{n}} c_{n} .
$$

Notice that, in case $m<n-1$, we have $g\left(c_{n-1}\right)=g\left(c_{m}\right)+\sum_{i=m}^{n-2} h_{i}>g\left(c_{m}\right)+$ $\frac{h_{m}}{2}$. Consequently, we have

$$
\begin{aligned}
& g\left(c_{n-1}\right)+\frac{h_{n-1}}{2} \geq g\left(c_{m}\right)+\frac{h_{m}}{2} \geq h(x)-r_{m}+\frac{h_{m}}{2} \stackrel{\text { by }}{>}(2) h(x) \\
& \quad \stackrel{\text { by }(7)}{\geq} g\left(c_{n}\right)-r_{n}-\frac{4(n+1) r_{n}}{a_{n}} c_{n} \stackrel{\text { by }(4)}{>} g\left(c_{n}\right)-\frac{h_{n-1}}{2}=g\left(c_{n-1}\right)+\frac{h_{n-1}}{2},
\end{aligned}
$$

which is a contradiction. 
Now, let $h$ be concave. Then we choose $1 \leq n<m, 1 \leq j<k \leq n$, $1 \leq l \leq m$ and $x_{1}, x_{2}, x \in[u, v]$ such that (6) holds.

Similarly as above, concavity of $h$ and our construction imply that

$$
\begin{aligned}
\frac{4(n+1) r_{n}}{a_{n}}=\frac{\left(g\left(b_{n}\right)+r_{n}\right)-\left(g\left(b_{n}\right)-r_{n}\right)}{\frac{a_{n}}{2(n+1)}} & \geq \frac{h\left(x_{2}\right)-h\left(x_{1}\right)}{x_{2}-x_{1}} \\
& \geq \frac{h(x)-h\left(x_{2}\right)}{x-x_{2}} \geq \frac{h(x)-g\left(b_{n}\right)-r_{n}}{x-x_{2}},
\end{aligned}
$$

and hence

$$
h(x)-g\left(b_{n}\right)-r_{n} \leq \frac{4(n+1) r_{n}}{a_{n}}\left(x-x_{2}\right) \leq \frac{4(n+1) r_{n}}{a_{n}}\left(1-b_{n}\right) .
$$

Notice that $g\left(b_{m}\right)=g\left(b_{n}\right)+\sum_{i=n}^{m-1} h_{i} \geq g\left(b_{n}\right)+\frac{h_{n}}{2}+\frac{h_{m-1}}{2}$. Thus we have

$$
\begin{aligned}
g\left(b_{n}\right)+\frac{h_{n}}{2} \leq g\left(b_{m}\right) & -\frac{h_{m-1}}{2} \leq h(x)+r_{m}-\frac{h_{m-1}}{2} \stackrel{\text { by }}{<}(2) \\
& \quad \leq h(x) \\
& \leq g\left(b_{n}\right)+r_{n}+\frac{4(n+1) r_{n}}{a_{n}}\left(1-b_{n}\right) \stackrel{\text { by }}{<}(3) g\left(b_{n}\right)+\frac{h_{n}}{2},
\end{aligned}
$$

which is a contradiction.

Claim 2. $f$ is differentiable at $\varphi(1)=(1,1)$ with $f^{\prime}(\varphi(1))=0$.

Proof. Recall that $f \equiv 0$ outside the balls $B_{j}^{(n)}(1 \leq j \leq n<\infty)$, and $f(\varphi(1))=0$. Let $P_{1}: \mathbb{R}^{2} \rightarrow \mathbb{R}$ denote the canonical projection on the first coordinate. For $x \in B_{j}^{(n)}$, we have $\|x-\varphi(1)\| \geq 1-P_{1}(x) \geq 1-\left(t_{j}^{(n)}+r_{n}\right)>$ $1-c_{n}>b_{n+1}-c_{n}=a_{n}$, and hence (by (2))

$$
0 \leq \frac{f(x)}{\|x-\varphi(1)\|} \leq \frac{f\left(z_{j}^{(n)}\right)}{a_{n}}=\frac{r_{n}}{n^{2} a_{n}}<\frac{1}{4(n+1) n^{2}} .
$$

Since $\varphi(1) \notin B_{j}^{(n)}(1 \leq j \leq n<\infty)$, it is easy to see that $\lim _{x \rightarrow \varphi(1)} \frac{f(x)}{\|x-\varphi(1)\|}=0$. We are done.

Convention. In what follows, given a mapping $F:[0, \ell] \rightarrow \mathbb{R}^{d}$ such that $F(\ell)=F(0)$, if necessary we consider $F$ extended to $\mathbb{R}$ as a (uniquely determined) $\ell$-periodic function. So, for instance, if the right derivative $F_{+}^{\prime}(t)$ exists at each $t \in[0, \ell)$ then the variation $V\left(F_{+}^{\prime},[0, \ell]\right)$ is calculated with $F_{+}^{\prime}(\ell):=F_{+}^{\prime}(0)$.

It is easy to see that this "circular variation" $V\left(F_{+}^{\prime},[0, \ell]\right)$ does not depend on shifts of the parameter. More precisely, if $F$ is extended periodically, $d \in \mathbb{R}$ and $G(t):=F(t+d)$, then $V\left(G_{+}^{\prime},[0, \ell]\right)=V\left(F_{+}^{\prime},[0, \ell]\right)$. 
We are going to prove that our (Lipschitz non-DC) function $f$ satisfies the following, a bit stronger variant of property $(\mathrm{C})$ :

$\left(\mathrm{C}^{\prime}\right)$ There exists a constant $c>0$ such that for each two-dimensional compact convex set $K \subset D$ and for each arc-length parametrization $r:[0, \ell] \rightarrow \partial K$ of $\partial K$ as a simple closed curve, and for $\Phi:=f \circ r$, we have (in the sense of our Convention):

(a) both one-sided derivatives $\Phi_{+}^{\prime}(t)$ and $\Phi_{-}^{\prime}(t)$ exist finite at each $t \in$ $[0, \ell]$

(b) $V\left(\Phi_{+}^{\prime},[0, \ell]\right) \leq c$ and $V\left(\Phi_{-}^{\prime},[0, \ell]\right) \leq c$.

To prove this, let $K \subset D$ and $r:[0, \ell] \rightarrow \partial K$ be as in $\left(\mathrm{C}^{\prime}\right)$. We can clearly assume that the parametrization $r$ is counterclockwise. The boundary $\partial K$ is the union of four parts:

$$
\partial K=G_{1} \cup G_{2} \cup G_{3} \cup G_{4},
$$

where:

- $G_{1}$ and $G_{3}$ are the graphs of a continuous convex and a continuous concave function, respectively, both defined on an interval $[\alpha, \beta] \subset(-2,2)$;

- $G_{2}, G_{4}$ are two (possibly degenerate) vertical line segments.

We can (and do) assume that $r$ "starts with the convex part", that is, for some $0<\ell_{1} \leq \ell_{2}<\ell_{3} \leq \ell$ we have

$$
r\left(\left[0, \ell_{1}\right]\right)=G_{1}, r\left(\left[\ell_{1}, \ell_{2}\right]\right)=G_{2}, r\left(\left[\ell_{2}, \ell_{3}\right]\right)=G_{3}, r\left(\left[\ell_{3}, \ell\right]\right)=G_{4} .
$$

(i) The "convex part" $G_{1}=r\left(\left[0, \ell_{1}\right]\right)$.

First suppose that there exists $t_{0} \in\left(0, \ell_{1}\right]$ such that $r\left(t_{0}\right)=\varphi(1)=(1,1)$. Given $\varepsilon \in\left(0, t_{0}\right)$, the set

$$
L_{\varepsilon}:=\left\{(n, j): n, j \in \mathbb{N}, j \leq n, r\left(\left[0, t_{0}-\varepsilon\right]\right) \cap B_{j}^{(n)} \neq \emptyset\right\}
$$

is finite. (This follows from the construction of the balls $B_{j}^{(n)}$, since $(1,1) \notin$ $r\left(\left[0, t_{0}-\varepsilon\right]\right)$.) Let $n_{\varepsilon}:=\max \left\{n \in \mathbb{N}:(n, j) \in L_{\varepsilon}\right.$ for some $\left.j \leq n\right\}$, and apply Claim 1 to choose an $m \leq n_{\varepsilon}$ such that $(n, j) \in L_{\varepsilon}$ for at most one $j$ whenever $n \leq n_{\varepsilon}$ and $n \neq m$. Let $P_{1}: \mathbb{R}^{2} \rightarrow \mathbb{R}$ be as in the proof of Claim 2. Since the projections

$$
I_{j}^{(n)}:=P_{1}\left(B_{j}^{(n)}\right), \quad(n, j) \in L_{\varepsilon},
$$

are finitely many pairwise disjoint compact subintervals of $(-2,2)$, there exist open intervals $U_{j}^{(n)},(n, j) \in L_{\varepsilon}$, such that $I_{j}^{(n)} \subset U_{j}^{(n)} \subset \overline{U_{j}^{(n)}} \subset(-2,2)$, $(n, j) \in L_{\varepsilon}$, and the intervals $\overline{U_{j}^{(n)}},(n, j) \in L_{\varepsilon}$, are pairwise disjoint.

Let $J_{j}^{(n)},(n, j) \in L_{\varepsilon}$, be relatively open subintervals of $\left[0, t_{0}-\varepsilon\right]$ such that

$$
K_{j}^{(n)}:=r^{-1}\left(B_{j}^{(n)}\right) \cap\left[0, t_{0}-\varepsilon\right] \subset J_{j}^{(n)} \subset \overline{J_{j}^{(n)}} \subset r^{-1}\left(U_{j}^{(n)} \times(-2,2)\right)
$$


for each $(n, j) \in L_{\varepsilon}$. (This is clearly possible since $P_{1} \circ r:\left[0, \ell_{1}\right] \rightarrow[\alpha, \beta]$ is an increasing homeomorphism.) Notice that

$$
f(x)=\max \left\{\frac{1}{n^{2}}\left(r_{n}-\left\|x-z_{j}^{(n)}\right\|\right), 0\right\}, \quad x \in U_{j}^{(n)} \times(-2,2),(n, j) \in L_{\varepsilon} .
$$

It is easy to see that the formula

$$
f(x)=\left(f(x)+\frac{1}{n^{2}}\left\|x-z_{j}^{(n)}\right\|\right)-\frac{1}{n^{2}}\left\|x-z_{j}^{(n)}\right\|, \quad x \in U_{j}^{(n)} \times(-2,2),
$$

is a representation of $f$ as a difference of two continuous convex functions. It follows from (5) that $f$ is controlled on each $U_{j}^{(n)} \times(-2,2),(n, j) \in L_{\varepsilon}$, by the function

$$
\gamma(x)=f(x)+\frac{2}{n^{2}}\left\|x-z_{j}^{(n)}\right\|=\max \left\{\frac{r_{n}}{n^{2}}+\frac{1}{n^{2}}\left\|x-z_{j}^{(n)}\right\|, \frac{2}{n^{2}}\left\|x-z_{j}^{(n)}\right\|\right\},
$$

which is Lipschitz with constant $\frac{2}{n^{2}}$. Denote $\Phi:=f \circ r$.

Fix $(n, j) \in L_{\varepsilon}$. If $J_{j}^{(n)}$ is open in $\mathbb{R}$, put $\mathcal{J}_{j}^{(n)}:=J_{j}^{(n)}$. Otherwise, since the length of $J_{j}^{(n)}$ is at most $\ell_{1}$, there exists an open (in $\mathbb{R}$ ) interval $\mathcal{J}_{j}^{(n)}$ of length smaller than $\ell$, such that $J_{j}^{(n)} \subset \mathcal{J}_{j}^{(n)}$ and $r\left(\overline{\mathcal{J}_{j}^{(n)}}\right) \subset U_{j}^{(n)} \times(-2,2)$. Then (we use Convention above for $r$ and $\Phi) K\left(r, \overline{\mathcal{J}_{j}^{(n)}}\right) \leq K_{0}^{\ell} r \leq 2 \pi$ by Lemma 1.1, and applying Proposition 2.1 we obtain

$$
K\left(\Phi, \overline{\mathcal{J}_{j}^{(n)}}\right) \leq(\operatorname{Lip}(f)+\operatorname{Lip}(\gamma)) K\left(r, \overline{\mathcal{J}_{j}^{(n)}}\right)+2 \operatorname{Lip}(\gamma) \operatorname{Lip}(r) \leq \frac{3}{n^{2}} 2 \pi+\frac{4}{n^{2}}
$$

Hence by [8, Proposition 3.4, p. 328], both derivatives $\Phi_{ \pm}^{\prime}$ exist at each point of $\mathcal{J}_{j}^{(n)}$, and we have

$$
V\left(\Phi_{ \pm}^{\prime}, J_{j}^{(n)}\right) \leq V\left(\Phi_{ \pm}^{\prime}, \mathcal{J}_{j}^{(n)}\right)=K\left(\Phi, \overline{\mathcal{J}_{j}^{(n)}}\right) \leq \frac{6 \pi+4}{n^{2}}
$$

Since $f \equiv 0$ outside all the balls $B_{j}^{(n)}, 1 \leq j \leq n<\infty$, we have that $\Phi \equiv 0$ outside the compact sets $K_{j}^{(n)},(n, j) \in L_{\varepsilon}$. Therefore both derivatives $\Phi_{ \pm}^{\prime}$ exist at each point of $\left(0, t_{0}-\varepsilon\right)$, and

$$
\Phi_{ \pm}^{\prime}(x)=0, \quad x \in\left(0, t_{0}-\varepsilon\right) \backslash \bigcup\left\{K_{j}^{(n)}:(n, j) \in L_{\varepsilon}\right\} .
$$

Choose relatively open subintervals $M_{j}^{(n)},(n, j) \in L_{\varepsilon}$, of $\left[0, t_{0}-\varepsilon\right]$ such that $K_{j}^{(n)} \subset M_{j}^{(n)} \subset \overline{M_{j}^{(n)}} \subset J_{j}^{(n)}$. Consider the extreme points of all the intervals $M_{j}^{(n)},(n, j) \in L_{\varepsilon}$, and index them in an increasing order to obtain the points

$$
0 \leq u_{1}<v_{1}<u_{2}<v_{2}<\cdots<u_{d}<v_{d} \leq t_{0}-\varepsilon,
$$

where $d=\operatorname{card} L_{\varepsilon}$. Then for each $(n, j) \in L_{\varepsilon}$ there is a unique $1 \leq k \leq d$ such that $\inf M_{j}^{(n)}=u_{k}$ and $\sup M_{j}^{(n)}=v_{k}$. 
Observe that both $\Phi_{ \pm}^{\prime}$ are identically null: on $\left(0, u_{1}\right]$ (if $\left.u_{1}>0\right)$; on $\left[v_{d}, t_{0}-\varepsilon\right.$ ) (if $v_{d}<t_{0}-\varepsilon$ ); on all intervals $\left[v_{k-1}, u_{k}\right], 2 \leq k \leq d$; on a neighborhood of any of the points $u_{k}, v_{k}(1 \leq k \leq d)$ that belongs to $\left(0, t_{0}-\varepsilon\right)$. Now, we can use this observation together with the additivity of variation and (1) to write

$$
\begin{aligned}
V\left(\Phi_{ \pm}^{\prime},\left(0, t_{0}-\varepsilon\right)\right) & =V\left(\Phi_{ \pm}^{\prime},\left(u_{1}, v_{d}\right)\right) \\
& =V\left(\Phi_{ \pm}^{\prime},\left(u_{1}, v_{1}\right]\right)+\sum_{k=2}^{d-1} V\left(\Phi_{ \pm}^{\prime},\left[u_{k}, v_{k}\right]\right)+V\left(\Phi_{ \pm}^{\prime},\left[u_{d}, v_{d}\right)\right) \\
& =\sum_{k=1}^{d} V\left(\Phi_{ \pm}^{\prime},\left(u_{k}, v_{k}\right)\right) \leq \sum_{(n, j) \in L_{\varepsilon}} V\left(\Phi_{ \pm}^{\prime}, J_{j}^{(n)}\right) .
\end{aligned}
$$

Thus, by (10) and the properties of $L_{\varepsilon}$ (see the text after its definition), we obtain

$$
\begin{aligned}
V\left(\Phi_{ \pm}^{\prime},\left(0, t_{0}-\varepsilon\right)\right) & \leq \sum_{n=1}^{n_{\varepsilon}} \frac{6 \pi+4}{n^{2}}+m \frac{6 \pi+4}{m^{2}} \\
& \leq(6 \pi+4)\left(1+\sum_{n=1}^{\infty} \frac{1}{n^{2}}\right)=: M .
\end{aligned}
$$

Consequently, $V\left(\Phi_{ \pm}^{\prime},\left(0, t_{0}\right)\right) \leq M$ since $\varepsilon \in\left(0, t_{0}\right)$ was arbitrary.

We claim that $V\left(\Phi_{ \pm}^{\prime},\left(0, \ell_{1}\right)\right) \leq M+1$. This is obvious if $t_{0}=\ell_{1}$. If $t_{0} \neq \ell_{1}$, we have $0<t_{0}<\ell_{1}, \Phi^{\prime}\left(t_{0}\right)=0$ (by Lemma 1.1 and Claim 2), and $\Phi \equiv 0$ on $\left[t_{0}, \ell_{1}\right)$. Since $\Phi$ is Lipschitz with constant 1 , we can use (1) to get

$$
V\left(\Phi_{ \pm}^{\prime},\left(0, \ell_{1}\right)\right)=V\left(\Phi_{ \pm}^{\prime},\left(0, t_{0}\right]\right) \leq V\left(\Phi_{ \pm}^{\prime},\left(0, t_{0}\right)\right)+1 \leq M+1,
$$

and we are done.

In case that $t_{0}$ does not exist, $G_{1}$ intersects at most finitely many balls $B_{j}^{(n)}$, and we can get the same estimate $V\left(\Phi_{ \pm}^{\prime},\left(0, \ell_{1}\right)\right) \leq M+1$ directly (as above with $\ell_{1}$ in place of $\left.t_{0}-\varepsilon\right)$.

(ii) The "concave part" $G_{3}=r\left(\left[\ell_{2}, \ell_{3}\right]\right)$.

This part can be treated in the very same way to obtain $V\left(\Phi_{ \pm}^{\prime},\left(\ell_{2}, \ell_{3}\right)\right) \leq$ $M+1$.

(iii) The two vertical segments $G_{2}=r\left(\left[\ell_{1}, \ell_{2}\right]\right)$ and $G_{4}=r\left(\left[\ell_{3}, \ell\right]\right)$.

By our construction (see the first inequality of (2)), each of the two vertical segments intersects at most one of the balls $B_{j}^{(n)}, 1 \leq j \leq n<\infty$. As in (9), for such $n$ we have $K\left(\Phi,\left[\ell_{1}, \ell_{2}\right]\right) \leq \frac{6 \pi+4}{n^{2}} \leq M+1$. As above, it follows that $V\left(\Phi_{ \pm}^{\prime},\left(\ell_{1}, \ell_{2}\right)\right) \leq M+1$ provided $\ell_{1}<\ell_{2}$; and in the same way $V\left(\Phi_{ \pm}^{\prime},\left(\ell_{3}, \ell\right)\right) \leq M+1$ provided $\ell_{3}<\ell$. 
(iv) Conclusion of the proof of $\left(\mathrm{C}^{\prime}\right)$.

For simplicity denote $\ell_{0}:=0$ and $\ell_{4}:=\ell$. Recall that $\Phi$ is Lipschitz with constant 1. Now, combining (1) with [8, Propositions 3.4, pp. 328-329], we obtain for $i=1,2,3,4$ that the derivatives $\Phi_{ \pm}^{\prime}\left(\ell_{i}\right)$ exist, and

$$
V\left(\Phi_{+}^{\prime},\left[\ell_{i-1}, \ell_{i}\right]\right) \leq V\left(\Phi_{+}^{\prime},\left(\ell_{i-1}, \ell_{i}\right)\right)+2 \leq M+3 .
$$

Thus

$$
V\left(\Phi_{+}^{\prime},[0, \ell]\right)=\sum_{i=1}^{4} V\left(\Phi_{+}^{\prime},\left[\ell_{i-1}, \ell_{i}\right]\right) \leq 4(M+3)
$$

and, symmetrically, $V\left(\Phi_{-}^{\prime},[0, \ell]\right) \leq 4(M+3)$. The proof is complete.

Acknowledgement. The authors thank the anonymous referee for very careful reading the manuscript. The research of the first author was partially supported by the MIUR, Italy, and by the GNAMPA - INdAM. The research of the second author was partially supported by the grant P201/15-08218S from the Grant Agency of Czech Republic.

\section{REFERENCES}

[1] M. Bačák, J. Borwein, On difference convexity of locally Lipschitz functions, Optimization 60 (2011), 961-978.

[2] J. Duda, Curves with finite turn, Czechoslovak Math. J. 58 (2008), 23-49.

[3] J. Duda, L. Veselý and L. Zajíček, On d.c. functions and mappings, Atti Sem. Mat. Fis. Univ. Modena 51 (2003), 111-138.

[4] I.M. Prudnikov, On the question of the representability of a function of two variables as the difference of convex functions, (Russian) Sibirsk. Mat. Zh. 55 (2014), no. 6, 1368-1380; translation in Sib. Math. J. 55 (2014), no. 6, 1116-1125.

[5] A.W. Roberts, E.D. Varberg, Convex Functions, Pure and Applied Mathematics, vol. 57, Academic Press, New York-London, 1973.

[6] H. Tuy, Convex Analysis and Global Optimization, 2nd ed., Springer Optimization and Its Applications 110, Springer, 2016.

[7] L. Veselý, L. Zajíček, Delta-convex mappings between Banach spaces and applications, Dissertationes Math. (Rozprawy Mat.) 289 (1989), 52 pp.

[8] L. Veselý and L. Zajíček, On vector functions of bounded convexity, Math. Bohem. 133 (2008), 321-335.

Dipartimento di Matematica, Università degli Studi, Via C. Saldini 50, 20133 MiLANO, ItALY

Charles University, Faculty of Mathematics and Physics, Sokolovská 83, 18675 PRAha 8, Czech Republic

E-mail address: libor.vesely@unimi.it

E-mail address: zajicek@karlin.mff.cuni.cz 